\title{
Exploring Diener's multi-dimensional conceptualization of well-being through network psychometrics
}

\author{
Giuntoli Laura ${ }^{1}$, Vidotto Giulio ${ }^{1}$ \\ ${ }^{1}$ Department of General Psychology, University of Padua, Padua, Italy
}

\author{
Laura Giuntoli \\ Department of General Psychology \\ University of Padua \\ Via Venezia, 8 \\ Padova (PD) 35131, Italy \\ E-mail: laura.giuntoli@phd.unipd.it \\ OrcID: 0000-0002-4833-5470
}

\begin{abstract}
Previous research has addressed the study of the well-being structure focusing on the separability among hedonic and eudaimonic dimensions by means of latent variable approaches. Diener's tripartite model of Subjective Well-Being (SWB) has often been identified with hedonic well-being, while flourishing (i.e., positive functioning) has been classified as a proxy for eudaimonic well-being. Instead of pursuing a theorydriven approach, the structure of well-being dimensions can be retrieved by means of network psychometrics that is a highly informative, data-driven approach that allows the model structure to spontaneously emerge from the relationships among indicators. Furthermore, we propose a strength centrality decomposition that is able to summarize the influence of a specific indicator within its dimension and between the other dimensions. Contrary to the theoretical conjecture that the separability among well-being dimensions can be grounded on a hedonic and eudaimonic distinction, our results point to a categorization of the Diener's well-being indicators based on the type of information they convey, that is global evaluation of life satisfaction, positive and negative affective experiences, and perceived positive functioning in life.
\end{abstract}

Keywords: SWB; Flourishing; Hedonic and Eudaimonic Well-being; Diener's model; Network Analysis; Expected Influence 


\section{Exploring Diener's multi-dimensional conceptualization of well-being through network psychometrics}

The interest in defining what happiness means have accompanied humanity since the dawn of philosophical thinking, and current well-being literature still largely revolve around the Hellenic concepts of hedonia and eudaimonia. According to the Socratic philosopher Aristippus, hedonia refers to the maximization of pleasure and the minimizing of suffering, while Aristotle contrasted such idea stating that the only way a human being can become happy is to pursuit eudaimonia which is living well, guided by values and virtues (Deci \& Ryan, 2008).

Measuring and understanding well-being is now an interdisciplinary field of study that operates at all levels of human kind systems, such as individuals, families, communities, organizations and countries (Huppert \& So, 2013). The pioneers of the scientific study of well-being were researchers in the field of economics and social indicators. During the seventies several scholars undermined the popular conception that quality of life can be adequately explained by objective life conditions such as income, marital status, age, education, etc. (e.g., Easterlin, 1974; Campbell, Converse, \& Rodgers, 1976; Wilson, 1967). Individuals' subjective judgements became the core elements of interest as researchers realized that people react differently to the same circumstances, and they evaluate their life conditions based on their expectations, values and previous experiences (Diener, Suh, Lucas, \& Smith, 1999). Initially, the researchers tended to treat well-being as a single, monolithic entity, often measured by a single global item in the form of "How happy do you feel in general?", but soon it became evident that well-being can be measured by separable components that show specific patterns of relations with different variables (Lucas, Diener, \& Suh, 1996).

According to Ed Diener (1984) the structure of Subjective Well-Being (SWB) can be conceptualized as having two fundamental components: The affective (experiential) component, measuring the presence of positive affect (PA) and the absence of negative affect (NA), and the cognitive (evaluative) component, measuring life satisfaction (LS). Consistent with its subjective 
focus, SWB is assessed using self-report measures to obtain separate scores of LS, PA and NA. Although Diener's (1984) tripartite model was subsequently expanded to cover domain-specific satisfaction appraisals, such as satisfaction with one's health or finances (e.g., Diener et al. 1999), the typical study of SWB involves the three components at a general level, based on the global evaluation of one's life, and on the positive and negative affective experiences at the trait level (Diener, 2008; Schimmack, 2008).

Since the publication of "Well-being: The foundations of hedonic psychology" (Kahneman, Diener, \& Schwarz, 1999), SWB was assimilated to the hedonic well-being. However, a more precise interpretation of hedonic well-being should only take into account the pleasant and unpleasant valence of affective experiences, while SWB also includes a cognitive assessment of subjective life conditions. Although the concept of SWB only partially overlaps with a hedonic definition of wellbeing, the hedonic label is mainly to distinguish it from the eudaimonic approach to the study of wellbeing. The development of the current eudaimonic definition of well-being emerged from the amalgam of different concepts of "good life", such as the actualization of human potentials (Waterman, 1993), the basic psychological needs for competence, autonomy and relatedness (Deci \& Ryan, 2000), and the psychological well-being dimensions (autonomy, environmental mastery, personal growth, positive relations with others, purpose in life and self-acceptance; Ryff, 1989; Ryff \& Keyes, 1995).

Pursuing an operationalization of eudaimonic well-being strictly pertinent to the theory could cause the risk of obtaining measures that are not comparable with the SWB outcome measures. There are two fundamental conceptual distinctions between the hedonic and eudaimonic perspectives: (a) Is the research interest oriented on the outcomes (i.e., happiness) or on the processes (i.e., eudaimonia)? And (b) is the criteria subjective or objective in the formulation of well-being indicators?

Eudaimonia is by definition a process which means living well by meeting objectively-valid needs and values to achieve well-being. As a consequence, eudaimonic dimensions can not be 
considered as direct indicators of well-being, but rather as determinants or distal factors that contribute to well-being. Shifting the object of the measurement from the processes to the outcomes and from an objective to a subjective perspective allows to operationalize the eudaimonic well-being as flourishing which is the perceived optimal functioning of the individuals. Flourishing is thus comparable to SWB because they are both measured at the same level and they can both be considered direct indicators of well-being. Nevertheless, numerous definitions exist of flourishing and different sets of dimensions have been taken into account by different authors. It is generally agreed that flourishing should integrate with both hedonic (positive emotions) and eudaimonic (e.g., meaning in life) components. For example, Seligman (2011) proposed that flourishing is constituted by five pillars, namely Positive emotions, Engagement, Relationships, Meaning and Accomplishment (PERMA), and Keyes (2002) defined flourishing as the positive pole of the mental health continuum, including several dimensions of social and psychological well-being, as well as positive emotions.

In a recent study Goodman, Disabato, Kashdan, \& Kauffman, (2018) compared Diener's SWB with Seligman's PERMA asking if the two constructs can be considered qualitatively different. The authors of the study showed that the two measures, despite being pigeonholed as hedonic (SWB) or eudaimonic (PERMA), end up measuring the same type of well-being therefore demonstrating that the dichotomy hedonic vs. eudaimonic does not represent a valid distinction between well-being components.

Instead of focusing on the distinction between hedonic and eudaimonic components, Diener's operationalization of well-being is focused on the distinction between affective and cognitive components, thus on the type of evaluation elicited by the items' content. It follows that flourishing should be measured separately from positive emotions because it does not (primarily) rely on affective information.

It remains unclear what the place of the flourishing construct is with respect to the affective and cognitive components of SWB. This paper is aimed to explore this research question by a data- 
driven approach, providing both a conceptual reasoning and an empirical basis for the validity of the Diener's multi-dimensional structure of well-being.

\section{Diener's operational definitions of well-being components}

\section{The cognitive component of Subjective Well-Being}

The measure of the cognitive component of well-being is based on LS, which represents a broad and reflective assessment of the individual's life (Diener, 2006). According to Evaluation Theory (Diener \& Lucas 2000), judgmental processes involve a comparison of one's perceived life circumstances with self-selected standards, influenced by subjective values, goals, motives and norms, as well as one's past history, personality and culture. Given the multiple relative standards on which respondents build their own judgements, it follows that individuals are likely to assign different weights to different life domains (Pavot \& Diener, 1993). That is why the authors of the Satisfaction With Life Scale (SWLS; Diener, Emmons, Larsen, \& Griffin, 1985) choose to assess LS using global items, allowing respondents to rate their life domains according to their own values in arriving at a subjective, global judgement of LS.

The SWLS have been deemed essentially one-dimensional (Slocum-Gori, Zumbo, Michalos, \& Diener, 2009), although the last two items do not fully load on the dominant latent factor. Indeed, their content (i.e., "So far I have gotten the important things I want in life" and "If I could live my life over, I would change almost nothing") push respondents to reflect above their present life satisfaction taking their past life experience as a standard of comparison.

\section{The affective component of Subjective Well-Being}

The affective component of the SWB is evaluated through PA and NA, which reflects the amount of pleasant and unpleasant feelings that people experience in their lives. Although early reports (e.g., Bradburn, 1969; Costa \& McCrae, 1980) proposed to combine PA and NA into an "affect balance" or "hedonic balance" score (i.e., PA minus NA), most researchers currently 
recommend that measures of PA and NA should not be combined into a single score because they are not inversely proportional and should not be considered as the two opposite poles of the same dimension (Diener \& Emmons, 1984).

One of the fundamental problems highlighted in measuring affect through self-reports, is that different people within and across cultures, identify and value different experiences in different ways given that evaluations of own emotional states are heavily influenced by identity, norms and beliefs (Diener, 1994; Tov \& Diener, 2007). With this in mind, Diener et al. (2010) constructed the Scale of Positive and Negative Experience (SPANE) to overcome some shortcuts of the popular Positive and Negative Affect Schedule (PANAS; Watson, Clark, \& Tellegen, 1988). First, the PANAS includes adjectives that do not represent real feelings ("strong," "alert," “determined," "active") or are infrequent (“inspired"). Second, the PANAS over-represents some feelings ("jittery," "nervous", "scared," and "afraid", to represent anxiety) at the expense of others (e.g., "sad" or "depressed" to represent depression which are absent). In general, the PANAS tends to over-represent states of high arousal, that are, anxiety for NA and enthusiasm for PA, whereas the states of low arousal are absent, both in terms of NA (i.e., depression) and PA (i.e., peace and serenity). This representation of PA and NA can be questionable, especially when applied to different cultures. In fact, while the emotional states characterized by high positive arousal are more emphasized in Western cultures, low-arousal feelings such as "calm" and "relaxed" are more desirable in Eastern cultures (Tsai, Knutson, \& Fung, 2006).

The SPANE represents a well balanced instrument, representing widely and equally both PA and NA. Using broad and general descriptors, such as "good", "positive", and "pleasant" for PA, and "bad", "negative", and "unpleasant" for NA, the SPANE allows the respondents to include the subtle nuances that color their subjective emotional experiences in their global evaluation. Specific feelings, both positive ("happy," "joyful," "contented") and negative ("sad," "afraid," "angry”), complete the full range of emotional states. Confirmatory factor studies conducted on the SPANE have identified two separate, correlated dimensions of PA and NA. 
Another remarkable distinction between the PANAS and the SPANE is that the former relies on the assessment of the intensity of feelings experienced by the respondents (i.e., "How much"), whereas the latter is based on a temporal frame (typically during the last month) in which respondents quantify the amount of time they passed in a particular feeling (i.e., "How often"). Indeed, the temporal frame seems more strongly related to well-being than the feeling's intensity (Diener, Colvin, Pavot, \& Allman, 1991; Jovanović, 2015).

\section{Flourishing}

Flourishing has been defined by Diener et al. (2010) as perceived optimal functioning and has been measured by the Flourishing Scale (FS). The FS taps eight aspects of human functioning identified by previous literature that can be roughly distinguished as social well-being (positive relationships with others, perception of the personal contribution to the well-being of others, being respected), and psychological well-being (meaning and purpose in life, perception of personal competence, self-acceptance, optimism). Importantly, the items are formulated to elicit the respondent's subjective evaluations on his own functioning.

From a structural point of view, the FS is conceived as a one-dimensional scale because confirmatory factor analyses repeatedly yielded a one factor solution across different studies (e.g., Giuntoli, Ceccarini, Sica, \& Caudek, 2017; Hone, Jarden, \& Schofield, 2013; Silva \& Caetano 2011). Contrary to other mainstream operationalizations of flourishing (e.g., Keyes, 2002; Seligman, 2011), the FS does not contain items assessing emotional well-being given that the affective components of SWB are measured separately by the SPANE.

\section{Methodological issues in retrieving the structure of well-being}

In the study of the well-being structure, most of the researchers have adopted reflective models (i.e., Confirmatory Factor Analysis, CFA) in which one or more latent factors cause the variability in 
the observed variables. Confirmatory factor models have been employed to test both the internal structure of the single well-being dimensions and the separability among them.

With respect to the internal structure of the specific Diener's constructs, several studies showed that the SWLS had a single underlying factor that represents LS, the SPANE had a twodimensional structure that distinguishes PA from NA, while the FS was described by a one-factor model although it covers distinct areas of life functioning. As regards to the FS internal structure, the CFA in some ways hides the information that can be extracted from the items' scale. In a CFA the covariances among items are constrained to zero and attributed to the influence of a latent factor. However, especially in the case of the FS where each item represents a distinct dimension, it is implausible that an underlying entity of optimal functioning causes optimism, having positive relationships, or meaning in life, rather, the reverse is more plausible. According to Keyes (2002) flourishing should be operationalized as an emerged condition based on the concept of a syndrome of symptoms of individuals' psychological and social functioning. For instance, sense of competence may activate other positive psychological features (e.g., meaning, self-acceptance and engagement), likely in circular, self-reinforcing ways. Thus, instead of modeling flourishing from a top-down perspective (i.e., by means of a confirmatory factor model), it could be more adequate to use a bottomup approach in which the FS items can be studied as active, interconnected indicators of positive functioning.

CFA has been the most widely used research method also in the case of the investigation on the separability among well-being dimensions. Although several studies have focused on the distinction between the affective and the cognitive components of SWB (e.g., Busseri \& Sadava, 2011), over time researchers became more interested in resolving the question if the well-being dimensions can be separated on the basis of their hedonic or eudaimonic content.

A number of studies suggests that hedonic and eudaimonic measures are distinguishable from one another (Compton, Smith, Cornish, \& Qualls, 1996; Keyes, Shmotkin, \& Ryff, 2002; Linley, Maltby, Wood, Osborne, \& Hurling, 2009), but it should be noted that these results could be due to 
the specific instruments employed. For example, Linley et al. (2009) used the Ryff's psychological well-being scales (Ryff \& Keyes, 1995) to assess eudaimonic well-being, and LS (SWLS; Diener et al., 1985), PA and NA (PANAS; Watson et al., 1988) to assess hedonic well-being. On the contrary, studies that assessed hedonic and eudaimonic well-being using different sets of measures failed to find structural differences among the two categories. For example, Disabato, Goodman, Kashdan, Short, \& Jarden (2016) did not consider affect measures and tested the separability of hedonic and eudaimonic well-being considering LS (SWLS; Diener et al., 1985), happiness (Subjective Happiness Scale; Lyubomirsky \& Lepper, 1999) and depression (Center for the Epidemiological Studies Depression Scale; Radloff, 1977) as hedonic measures on the one side, and the six subscales of Ryff's psychological well-being (Ryff \& Keyes 1995) as measures of eudaimonic well-being on the other.

At present, therefore, because the latent variable approach requires an a-priori specification of the models and because there is not a common understanding of which measures tap hedonic or eudaimonic contents, the validity of the separability among well-being components using the hedonic and eudaimonic categories is doubtful. Instead, it could be useful to address this problem by using a data-driven approach to explore what kind of categories emerge from the relationships among different type of indicators.

Given the limitations of CFA to resolve specific problems such as modeling single indicators as separate dimensions (the case of the FS) and identifying categories of measures without specify apriori hypotheses (the case of hedonic vs. eudaimonic components), network analysis (Epskamp, Rhemtulla, \& Borsboom, 2017) could represent an insightful step ahead in the evaluation and generation of well-being models, and may offer a constructive way forward to suggest the presence of naturally occurring categories.

\section{Overview of network psychometrics modeling}

Network psychometrics is a new field of statistical modeling which focuses on the estimation of undirected network models (Epskamp, Maris, Waldorp, \& Borsboom, 2018). A network is 
composed of a set of nodes (any kind of entity) and a set of edges (any kind of relationship) which connect these nodes. Differently from other types of network models, such as social networks, the peculiarity of a psychometric network is that it can be used to estimate edge weights, and thus it is particularly suitable to represent a psychological construct in which nodes are questionnaire items, while the edges connecting them are partial correlations (i.e., the covariation displayed between two nodes is conditionally independent of all the other nodes in the network).

To avoid over-fitting and to make the model easier to interpret removing redundant variables that do not add any information, partial correlations are subjected to a Graphical Least Absolute Shrinkage and Selection Operator (GLASSO; Epskamp \& Fried, 2018; Friedman, Hastie, \& Tibshirani, 2008). GLASSO regularization operates by controlling the tuning parameter $\lambda$ to estimate a sparse network in which the spurious partial correlations are shrunk to zero. An efficient method to select the best value for $\lambda$ is by minimizing the Extended Bayesian Information Criterion (EBIC; Chen \& Chen, 2008; Foygel \& Drton, 2010). In this way, EBIC GLASSO estimation produces a network solution with an optimal balance between sparsity and representativity of the data.

Edges connecting nodes can differ in their strength, indicating if a relationship is strong (visualized as thicker edges) or weak (visualized as less saturated edges), and positive (green edges) or negative (red edges). The properties of a network structure can be summarized by the centrality metrics: Strength is the sum of a node's edge weights; Closeness is the inverse of the sum of the shortest paths between a specific node and all the other nodes; Betweenness is the total number of shortest paths that pass through a node. The more a node is central, the more it has an influence on all the other nodes, meaning that it could be considered a particularly important indicator of the construct. Robinaugh, Millner, \& McNally (2016) showed that when strength centrality is calculated using absolute weights it become less predictive of observed node influence as the proportion of negative edges increases. Therefore, they proposed to calculate expected influence (EI) that it is equal to the node strength except that it takes into account also the negative or positive sign of the edge weight's value. When in a network can be identified sub-communities of nodes, bridge centrality 
metrics (Jones, 2017) are useful to identify which nodes are important in cross-communities connections. Bridge-EI is calculated as the sum of the values of all the edges that connect a node with all the nodes that are not in the same community, with higher values meaning that a node has a greater influence on nodes of the other nodes' communities overall.

Dealing with psychological constructs, it can also be important to interpret the edges in terms of explained variance, thus Haslbeck and Waldorp (2018) recently proposed to analyze the predictability of nodes as an absolute measure of centrality, that is, how much of the variance of a node is explained by the edges connected to that specific node. Noteworthy, predictability across nodes can be interpreted as a determination index, with strong mutual interactions between nodes defining high predictability of the network. Conversely, when predictability is low, unexplained variance could derive from other variables that were not included in the network.

A recent advancement in network psychometric is Exploratory Graph Analysis (EGA; Golino \& Epskamp, 2017) that aims to uncover latent dimensions in network models by using a clustering algorithm for weighted networks (walktrap; Pons \& Latapy, 2006). Indeed, nodes may cluster together forming tidy connected sub-networks. It has been mathematically demonstrated that clusters in networks equal latent variables (Chandrasekaran, Parrilo, \& Willsky, 2012, Epskamp et al., 2017): When clusters in a network are unconnected they are a result of orthogonal factors, whereas when the clusters are connected they represents correlated factors (Golino \& Epskamp 2017).

An advantage of using network psychometrics and EGA is that they are data-driven approaches. With respect to CFA there is no need of an a-priori theory to specify the model. This is a particularly interesting approach to answer the question on the dimensionality of well-being, because instead of testing the alternative hypotheses of multi-dimensionality vs. one-dimensionality of the well-being construct, we can "let the data speak by themselves". Furthermore, comparing to other extraction techniques in recovering the correct number of factors, EGA demonstrated an accuracy of $100 \%$, far exceeding the performance, for example, of exploratory factor analysis (mean 
accuracy $=10-49 \%$ ) and CFA using the Akaike information criterion (mean accuracy $=74 \%$; Golino \& Demetriou, 2017; Keith, Caemmerer, \& Reynolds, 2016).

In the light of this promising approach, this study is aimed to give a different methodological perspective on the interpretation of the well-being dimensionality. Network analysis is a powerful and intuitive statistical tool that could complement previous studies relying on latent factor approach improving our understanding of the complex relationships between the different facets of well-being.

\section{The current study}

Given the well-known tripartite structure of SWB, the present study aims to empirically explore the validity of a quadripartite model of well-being that also includes the Diener's operational definition of flourishing. A prerequisite to compare the SWB dimensions with flourishing is that they need to be measured at the same level (i.e., outcome measures), indeed, the SWLS, the SPANE and the FS are all based on the subjective evaluations of the individuals and they are all time-framed over a period of one month.

Through network psychometrics it is possible to explore two orders of research questions. First, the estimated network model let the well-being structure spontaneously emerge without the need to specify a-priori hypotheses on the model structure, for example, whether the items cluster together according to the four dimensions proposed by Diener, or if flourishing should also encompass positive affect as stated by Seligman (2011) and Keyes (2002). Second, the FS taps eight dimensions that are represented by eight single items. Whereas confirmatory factor studies failed to catch the FS multi-dimensionality, because at least three observed variables are needed to contribute to a factor (Bollen, 1989), network modeling does not bring into play latent factors and allows to examine the specific contribution of each of the eight FS items. 


\section{Method}

\section{Participants}

Participants were enrolled by means of adverts posted on Italian websites and social network groups. A total sample of 2392 respondents completed the well-being questionnaires. Average age was 27.8 years $(S D=8.9)$ for females $(N=1882)$ and 29.5 years $(S D=11.1)$ for males $(\mathrm{N}=510)$. Participation in this study was anonymous and on a voluntary basis. All the participants provided their electronic informed consent prior to completing the online aforementioned Diener's and colleagues scales, namely the SWLS, the SPANE, and the FS.

\section{Measures}

The SWLS (Diener et al., 1985) is a 5-item measure of the cognitive component of SWB. The respondents use a 7-point Likert-type scale (from $1=$ "strongly disagree" to 7 = "strongly agree") to rate their general life satisfaction. Total scores range from 5 to 25, with higher scores reflecting greater satisfaction with life.

The SPANE (Diener et al., 2010) is a 12-item two-dimensional scale that measures the affective component of SWB. Six items assess positive affect (SPANE-P) and six items assess negative affect (SPANE-N). Respondents rate how often they had experienced the feelings indicated by each item over the past four-weeks by means of a 5-point Likert-type scale (from 1 = "very rarely or never" to $5=$ "very often or always"). The total SPANE-P and SPANE-N scores range from 6 to 30 and are scored separately as they measure independent feelings.

The FS (Diener et al., 2010) measures psycho-social functioning in different areas of life by means of eight items on a 7-point Likert-type scale (from $1=$ "strongly disagree" to 7 = "strongly agree"). Total scores range from 8 to 56 , with higher scores indicating optimal functioning.

In Table 1 are detailed the items included in each well-being dimension with the assigned label used for the subsequent analyses. 
Table 1

Items indexed in the Diener's well-being scales.

\begin{tabular}{ccl}
\hline Dimension & Node label & Item content \\
\hline & $\mathrm{f} 1$ & I lead a purposeful and meaningful life \\
Flourishing & $\mathrm{f} 2$ & My social relationships are supportive and rewarding \\
(F) & $\mathrm{f} 4$ & I am engaged and interested in my daily activities \\
& $\mathrm{f} 5$ & I am competent and capable in the activities that are important to me \\
& $\mathrm{f} 6$ & I am a good person and live a good life \\
& $\mathrm{f} 7$ & I am optimistic about my future \\
f8 & People respect me \\
\hline \multirow{3}{*}{ Life Satisfaction } & ls1 & In most ways my life is close to my ideal \\
(LS) & ls2 & The conditions of my life are excellent \\
& ls3 & I am satisfied with my life \\
& ls4 & So far I have gotten the important things I want in life \\
& ls5 & If I could live my life over, I would change almost nothing \\
\hline Positive Affect & pa1 & Positive \\
(PA) & pa2 & Good \\
& pa3 & Pleasant \\
& pa4 & Happy \\
& pa5 & Joyful \\
& pa6 & Contented \\
\hline & na1 & Negative \\
Negative Affect & na2 & Bad \\
(NA) & na3 & Unpleasant \\
& na4 & Sad \\
& na5 & Afraid \\
& na6 & Angry
\end{tabular}

Note: Flourishing indicators are identified by the label " $\mathrm{f}$ " and a number ranging from 1 to 8 , referring to the 8 items of the FS; Life Satisfaction indicators are identified by the label "ls" and a number ranging from 1 to 5, referring to the 5 items of the SWLS; Each item from the SPANE is designated by a number ranging from 1 to 6 accompanied either by the label "pa" or "na", representing Positive Affect and Negative Affect, respectively.

\section{Statistical analyses}

We conducted our analyses with the R software (version 3.6.0, R Core Team, 2019). A EBIC GLASSO network model with all the 25 well-being items was estimated and visualized via the Rpackages qgraph (version 1.6.2; Epskamp, Cramer, Waldorp, Schmittmann, \& Borsboom, 2012) and bootnet (version 1.2.2; Epskamp, 2019), while the dimensionality of the model was determined by means of the EGA package for R (version 0.2; Golino \& Epskamp, 2017). 
The analysis of the network structure relied on the concept of strength centrality. The formula to calculate the strength ( $\mathrm{s}$; or Expected Influence, $\mathrm{EI}$ ) of a node $\mathrm{V}_{\mathrm{i}}$ is defined as:

$$
\mathrm{EI}_{\mathrm{i}}=\sum_{\mathrm{j}=1}^{\mathrm{N}} \mathrm{w}_{\mathrm{ij}}, \quad \forall \mathrm{V}_{\mathrm{j}} \text { where } \mathrm{j} \neq \mathrm{i}
$$

where $\mathrm{N}$ is the total number of the nodes in the network, and $\mathrm{w}_{\mathrm{ij}}$ is the element in the weights matrix that corresponds to the row $\mathrm{i}$, namely the node of interest $\mathrm{V}_{\mathrm{i}}$, and to the column(s) j, representing all the other nodes $\left(\mathrm{V}_{\mathrm{j}}\right)$ in the network except $\mathrm{V}_{\mathrm{i}}$.

Another important strength centrality metric proposed by the current state of the art in network psychometrics is bridge-EI (Jones, 2017) that considers the influence of a node on the rest of the network except with the nodes' community it belongs to $\left(\mathrm{C}_{\mathrm{k}}\right)$ and it is defined as:

$$
\text { bridge-EI } I_{i}^{k}=\sum_{j=1}^{N} w_{i j}, \quad \forall V_{j} \notin \mathrm{C}_{k} \text { where } j \neq i
$$

Dealing with a multi-dimensional network we also deemed it important to consider the influence of a node with respect to specific dimensions, thus we additionally present two new measures of nodes' importance that decompose EI and bridge-EI. Given that EI represents the total effect of a node on all the other nodes in the network, it can be decomposed in bridge-EI (i.e., the effect of a node on the network, excluded the nodes belonging to its own dimension), and in the strength of the connections of a node with the nodes belonging to the same dimension, here named "within-EI" (to denote the EI of a node within the same community $\mathrm{C}_{\mathrm{k}}$ ). The within-EI of a node Vi is defined as:

$$
\text { within-EI } I_{i}^{k}=\sum_{j=1}^{N} w_{i j}, \quad \forall V_{j} \in C_{k} \text { where } j \neq i
$$

Furthermore, when there are more than two communities in a network it is useful to decompose the importance of a node separately for each of the other nodes communities. Instead of considering globally bridge-EI, the strength of the connections of a node $\mathrm{V}_{\mathrm{i}}$ with a specific dimension 
other than its own, is here named "between-EI" (to denote the EI of a node $\mathrm{V}_{\mathrm{i}}$ between communities) and it is defined as:

$$
\text { between-EI } \mathrm{I}_{\mathrm{i}}^{\mathrm{k}_{1} \mathrm{k}_{2}}=\sum_{\mathrm{j}=1}^{\mathrm{N}} \mathrm{w}_{\mathrm{ij}}, \quad \forall \mathrm{V}_{\mathrm{j}} \in \mathrm{C}_{\mathrm{k}_{2}} \text { where } \mathrm{V}_{\mathrm{i}} \in \mathrm{C}_{\mathrm{k}_{1}} \& \mathrm{C}_{\mathrm{k}_{1}} \neq \mathrm{C}_{\mathrm{k}_{2}}
$$

where the superscript $\mathrm{k}_{1}$ indicates the belonging community of the node $\mathrm{V}_{\mathrm{i}}$, whereas the superscript $\mathrm{k}_{2}$ indicates a specific community of nodes other than the community of node $\mathrm{V}_{\mathrm{i}}$.

Thus, bridge-EI corresponds to the sum of all the between-EI indices, while EI corresponds to the sum of within-EI plus bridge-EI.

Similarly to expected influence, nodes predictability depends on the number and the strength of the edges a node is connected to, but it has the advantage to provide an absolute index of explained variance $\left(\mathrm{R}^{2}\right)$ that ranges from 0 (meaning that a node cannot be predicted by its neighbors) to 1 (meaning that a node can be perfectly predicted by its neighbors). The nodes predictability was computed via the $m g m$ R package (version 1.2-6; Haslbeck \& Waldorp, 2016).

\section{Results}

\section{Network dimensionality}

The weights matrix (Table 2) of the 25 well-being items was obtained by using LASSO regularization with EBIC model selection and the resulting network is shown in Figure 1. The well-being items formed four tightly connected groups, with the items clustering together according to their theoretical dimension. In fact, the thicker edges were those connecting the items relying on the same dimension. These almost fully connected sub-networks can be interpreted as the effect of the unmodeled latent variables on the items. In a multi-dimensional network, the more central items would be mainly connected to other items associated with the respective domain.

Although positive and negative affect are deemed as separate dimensions, their items showed strong ties, especially the opposite general items such as positive-negative (pa1-na1), good-bad (pa2na2) and pleasant-unpleasant (pa3-na3). Consistently with the SWB conceptualization, PA and NA 
tended to group together into an affective component, whereas LS formed another cluster, namely the cognitive component. It is interesting to note that flourishing constituted a separate cluster, and that its items were connected both with the cognitive and the affective components' items.

The EGA confirmed that four sub-networks were identified in the well-being network structure, namely flourishing, LS, PA and NA. Confirmatory factor analysis showed that the structure of the data suggested by EGA attained an adequate fit to the data $\left[\chi^{2}(269)=2295.96\right.$; CFI $=.994$; $\mathrm{RMSEA}=0.056 ; \mathrm{NFI}=0.993 ; \mathrm{NNFI}=0.993]$. 
Table 2

Estimated weights matrix using EBIC GLASSO

\begin{tabular}{|c|c|c|c|c|c|c|c|c|c|c|c|c|c|c|c|c|c|c|c|c|c|c|c|c|c|}
\hline & f1 & $\mathrm{f} 2$ & $\mathrm{f} 3$ & $\mathrm{f} 4$ & $\mathrm{f} 5$ & f6 & f7 & f8 & 1s 1 & $1 \mathrm{~s} 2$ & ls 3 & 1s4 & ls5 & pa1 & pa2 & pa3 & pa4 & pa5 & pa6 & na1 & na2 & na3 & na4 & na5 & na6 \\
\hline f1 & .00 & .14 & .35 & .00 & .06 & .06 & .18 & .00 & .07 & .00 & .00 & .00 & .00 & .04 & .00 & .00 & .00 & .00 & .07 & .05 & .00 & .00 & .00 & .00 & .00 \\
\hline $\mathrm{f} 2$ & .14 & .00 & .18 & .21 & -.09 & .00 & .00 & .26 & .00 & .00 & .00 & .00 & .00 & .00 & .00 & .00 & .00 & .00 & .00 & .00 & .00 & .00 & .00 & .00 & .00 \\
\hline f3 & .35 & .18 & .00 & .18 & .17 & .00 & .00 & .00 & .00 & .00 & .08 & .00 & .00 & .00 & .00 & .00 & .00 & .00 & .09 & .00 & .00 & .00 & .00 & .00 & .00 \\
\hline f4 & .00 & .21 & .18 & .00 & .25 & .19 & .00 & .00 & .00 & .00 & .00 & .00 & .00 & .00 & .00 & .00 & .00 & .00 & .00 & .00 & .00 & .00 & .00 & .00 & .00 \\
\hline f5 & .06 & -.09 & .17 & .25 & .00 & .28 & .00 & .18 & .00 & .00 & .00 & .00 & .00 & .00 & .00 & .00 & .00 & .00 & .00 & .00 & .00 & .00 & .00 & .00 & .00 \\
\hline f6 & .06 & .00 & .00 & .19 & .28 & .00 & .20 & .15 & .00 & .07 & .00 & .00 & .00 & .00 & .00 & .00 & .00 & .00 & .00 & .00 & -.06 & .00 & .00 & .00 & .00 \\
\hline f7 & .18 & .00 & .00 & .00 & .00 & .20 & .00 & .14 & .08 & .00 & .04 & .00 & .00 & .11 & .00 & .00 & .00 & .00 & .00 & -.08 & .00 & .00 & .00 & .00 & .00 \\
\hline f8 & .00 & .26 & .00 & .00 & .18 & .15 & .14 & .00 & .05 & .00 & .00 & .00 & .00 & .00 & .00 & .00 & .00 & .00 & .00 & .00 & .00 & .00 & .00 & .00 & .00 \\
\hline ls1 & .07 & .00 & .00 & .00 & .00 & .00 & .08 & .05 & .00 & .22 & .30 & .20 & .18 & .00 & .00 & .00 & .00 & .00 & .00 & .00 & .00 & .00 & .00 & .00 & .00 \\
\hline 1s2 & .00 & .00 & .00 & .00 & .00 & .07 & .00 & .00 & .22 & .00 & .33 & .00 & .00 & .00 & .00 & .00 & .00 & .00 & .00 & .00 & .00 & .00 & .00 & .00 & .00 \\
\hline 1s3 & .00 & .00 & .08 & .00 & .00 & .00 & .04 & .00 & .30 & .33 & .00 & .30 & .18 & .00 & .00 & .00 & .00 & .00 & .00 & .00 & .00 & .00 & -.07 & .00 & .00 \\
\hline ls4 & .00 & .00 & .00 & .00 & .00 & .00 & .00 & .00 & .20 & .00 & .30 & .00 & .19 & .00 & .00 & .00 & .00 & .00 & .05 & .00 & .00 & .00 & .00 & .00 & .00 \\
\hline 1s5 & .00 & .00 & .00 & .00 & .00 & .00 & .00 & .00 & .18 & .00 & .18 & .19 & .00 & .00 & .00 & .00 & .04 & .00 & .00 & .00 & .00 & .00 & .00 & .00 & .00 \\
\hline pa1 & .04 & .00 & .00 & .00 & .00 & .00 & .11 & .00 & .00 & .00 & .00 & .00 & .00 & .00 & .22 & .17 & .25 & .08 & .11 & -.33 & .08 & .00 & .00 & -.05 & .00 \\
\hline pa2 & .00 & .00 & .00 & .00 & .00 & .00 & .00 & .00 & .00 & .00 & .00 & .00 & .00 & .22 & .00 & .30 & .00 & .00 & .06 & .11 & -.29 & .00 & .08 & .00 & .00 \\
\hline pa3 & .00 & .00 & .00 & .00 & .00 & .00 & .00 & .00 & .00 & .00 & .00 & .00 & .00 & .17 & .30 & .00 & .18 & .18 & .06 & .00 & .11 & -.15 & .00 & .00 & .00 \\
\hline pa4 & .00 & .00 & .00 & .00 & .00 & .00 & .00 & .00 & .00 & .00 & .00 & .00 & .04 & .25 & .00 & .18 & .00 & .35 & .15 & .00 & .00 & .00 & -.13 & .00 & .00 \\
\hline pa5 & .00 & .00 & .00 & .00 & .00 & .00 & .00 & .00 & .00 & .00 & .00 & .00 & .00 & .08 & .00 & .18 & .35 & .00 & .16 & -.04 & .00 & .00 & .00 & .00 & .00 \\
\hline pa6 & .07 & .00 & .09 & .00 & .00 & .00 & .00 & .00 & .00 & .00 & .00 & .05 & .00 & .11 & .06 & .06 & .15 & .16 & .00 & -.08 & .06 & .00 & .00 & .00 & .00 \\
\hline na1 & .05 & .00 & .00 & .00 & .00 & .00 & -.08 & .00 & .00 & .00 & .00 & .00 & .00 & -.33 & .11 & .00 & .00 & -.04 & -.08 & .00 & .14 & .27 & .26 & .13 & .07 \\
\hline na2 & .00 & .00 & .00 & .00 & .00 & -.06 & .00 & .00 & .00 & .00 & .00 & .00 & .00 & .08 & -.29 & .11 & .00 & .00 & .06 & .14 & .00 & .34 & .10 & .00 & .18 \\
\hline na3 & .00 & .00 & .00 & .00 & .00 & .00 & .00 & .00 & .00 & .00 & .00 & .00 & .00 & .00 & .00 & -.15 & .00 & .00 & .00 & .27 & .34 & .00 & .12 & .09 & .10 \\
\hline na4 & .00 & .00 & .00 & .00 & .00 & .00 & .00 & .00 & .00 & .00 & -.07 & .00 & .00 & .00 & .08 & .00 & -.13 & .00 & .00 & .26 & .10 & .12 & .00 & .23 & .20 \\
\hline na5 & .00 & .00 & .00 & .00 & .00 & .00 & .00 & .00 & .00 & .00 & .00 & .00 & .00 & -.05 & .00 & .00 & .00 & .00 & .00 & .13 & .00 & .09 & .23 & .00 & .00 \\
\hline na6 & .00 & .00 & .00 & .00 & .00 & .00 & .00 & .00 & .00 & .00 & .00 & .00 & .00 & .00 & .00 & .00 & .00 & .00 & .00 & .07 & .18 & .10 & .20 & .00 & .00 \\
\hline
\end{tabular}

Note: The weights matrix is symmetric because the network is undirected and each edge weight is represented twice, above and below the main diagonal. A value of zero in the weights matrix encodes no connection between two nodes. 


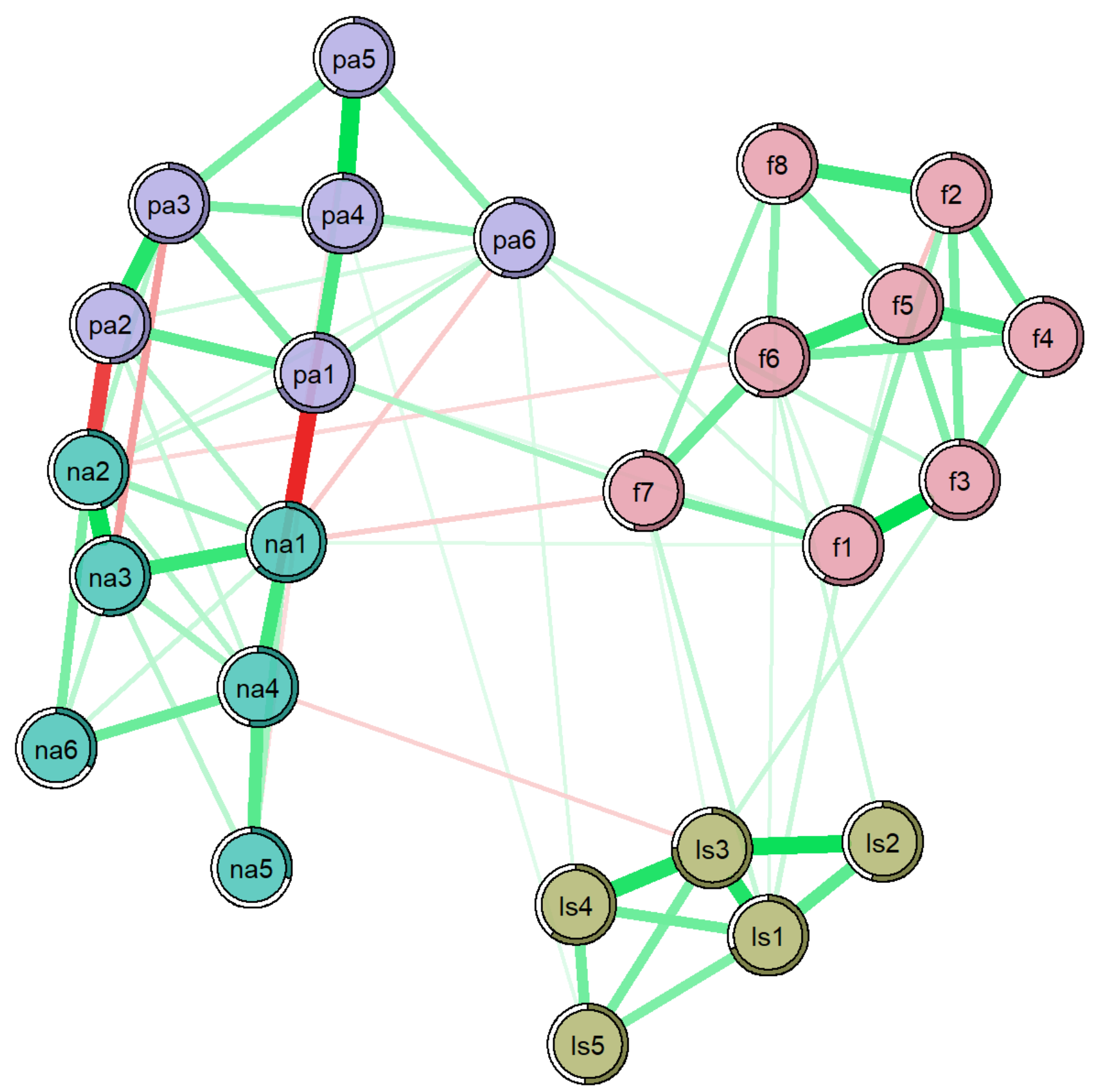

Figure 1. EBIC GLASSO network model of well-being. The nodes, representing the items, are colored according to their respective dimension (the items are detailed in Table 1).

\section{Nodes' importance}

Global expected influence and nodes predictability. The analyses of expected influence (Table 3) showed that the nodes "I am satisfied with my life" (ls3) and "In most ways my life is close to my 
ideal" (ls1) exhibited the highest levels of EI compared to all the other nodes in the network. Notably, these two items represent the most general level of abstraction regarding the evaluation of one's own life.

Bridge-EI provides a global synthesis to interpret the cross-dimensional connections among nodes. Within the flourishing dimension, nodes denoting purpose in life (f1), engagement in daily activities (f3), and optimism (f7) exhibited the highest levels of bridge-EI on all the other dimensions. The node "In most ways my life is close to my ideal" (1s1) was the LS indicators exhibiting the highest level of bridge-EI. Among the affective component's nodes, the higher bridge-EI was shown by the node "Contented" (pa6).

Reflecting expected influence, nodes predictability (see Table 3) ranged between .29 (na5) and .75 (ls3), with a mean predictability across all nodes of 0.55 , indicating an explained variance of the network model of 55\%. As a final remark, the node 1s3 ("I am satisfied with my life") showed the highest predictability $\left(\mathrm{R}^{2}=.75\right)$, as well the higher expected influence $(\mathrm{EI}=1.18)$. This could be interpreted as the effect of the ls 3 semantic content as subordinate to all the other items in the network. In other words, the global judgement over one's own life mostly emerge from the contribute of the interacting facets of well-being, that is consistent with the definition of the evaluation of life as a whole relying on multiple terms of comparison.

Flourishing internal structure. Flourishing items tap different areas of life, but in general they were positively associated to each other. Flourishing indicators showed high within-EI indices (Table 3), supporting the good internal consistency of the FS.

The specific connections among nodes allowed to unveil the reciprocal influence between functioning in different aspects of life. For example, f2 ("My social relationships are supportive and rewarding") and f5 ("I am competent and capable in the activities that are important to me") were negatively associated, but they were positively connected through the node f4 ("I actively contribute to the happiness and well-being of others"). This pattern can be interpreted, for instance, as follows: having high self-efficacy is not necessarily a direct predictor of having positive relationships, but 
when a capable person also orientates his or her actions to social contribution, he or she also experiences having positive social relationships. The indirect positive association between $\mathrm{f} 2$ and $\mathrm{f} 5$ was also supported by the nodes f3 ("I am engaged and interested in my daily activities") and f8 ("People respect me").

Table 3

Nodes metrics describing the network structure

\begin{tabular}{|c|c|c|c|c|c|c|c|c|c|c|c|}
\hline \multirow[t]{2}{*}{ Node } & \multicolumn{4}{|c|}{ Within-EI } & \multicolumn{4}{|c|}{ Between-EI } & \multirow[t]{2}{*}{ Bridge-EI } & \multirow[t]{2}{*}{ EI } & \multirow[t]{2}{*}{$\mathrm{R}^{2}$} \\
\hline & $\mathrm{F}$ & LS & $\mathrm{PA}$ & NA & $\mathrm{F}$ & LS & $\mathrm{PA}$ & NA & & & \\
\hline f1 & 0.790 & & & & & 0.075 & 0.107 & 0.046 & 0.227 & 1.017 & 0.582 \\
\hline $\mathrm{f} 2$ & 0.700 & & & & & 0.000 & 0.000 & 0.000 & 0.000 & 0.700 & 0.507 \\
\hline $\mathrm{f} 3$ & 0.884 & & & & & 0.076 & 0.092 & 0.000 & 0.168 & 1.052 & 0.631 \\
\hline $\mathrm{f} 4$ & 0.826 & & & & & 0.000 & 0.000 & 0.000 & 0.000 & 0.826 & 0.501 \\
\hline f5 & 0.851 & & & & & 0.000 & 0.000 & 0.000 & 0.000 & 0.851 & 0.519 \\
\hline f6 & 0.881 & & & & & 0.072 & 0.000 & -0.063 & 0.010 & 0.891 & 0.549 \\
\hline f7 & 0.527 & & & & & 0.124 & 0.112 & -0.076 & 0.160 & 0.688 & 0.542 \\
\hline f8 & 0.739 & & & & & 0.053 & 0.000 & 0.000 & 0.053 & 0.793 & 0.449 \\
\hline ls1 & & 0.905 & & & 0.209 & & 0.000 & 0.000 & 0.209 & 1.114 & 0.694 \\
\hline 1s2 & & 0.557 & & & 0.072 & & 0.000 & 0.000 & 0.072 & 0.629 & 0.546 \\
\hline 1s 3 & & 1.126 & & & 0.119 & & 0.000 & -0.069 & 0.049 & 1.176 & 0.751 \\
\hline ls4 & & 0.698 & & & 0.000 & & 0.053 & 0.000 & 0.053 & 0.751 & 0.610 \\
\hline 1s5 & & 0.556 & & & 0.000 & & 0.043 & 0.000 & 0.043 & 0.599 & 0.512 \\
\hline pa1 & & & 0.830 & & 0.153 & 0.000 & & -0.300 & -0.147 & 0.683 & 0.687 \\
\hline pa2 & & & 0.581 & & 0.000 & 0.000 & & -0.100 & -0.100 & 0.481 & 0.498 \\
\hline pa3 & & & 0.893 & & 0.000 & 0.000 & & -0.040 & -0.040 & 0.853 & 0.595 \\
\hline pa4 & & & 0.935 & & 0.000 & 0.043 & & -0.130 & -0.086 & 0.848 & 0.663 \\
\hline pa5 & & & 0.762 & & 0.000 & 0.000 & & -0.041 & -0.041 & 0.721 & 0.581 \\
\hline pa6 & & & 0.539 & & 0.158 & 0.053 & & -0.021 & 0.189 & 0.728 & 0.554 \\
\hline na1 & & & & 0.867 & -0.030 & 0.000 & -0.339 & & -0.370 & 0.498 & 0.634 \\
\hline na2 & & & & 0.759 & -0.063 & 0.000 & -0.041 & & -0.104 & 0.655 & 0.444 \\
\hline na3 & & & & 0.925 & 0.000 & 0.000 & -0.146 & & -0.146 & 0.779 & 0.530 \\
\hline na4 & & & & 0.915 & 0.000 & -0.069 & -0.050 & & -0.120 & 0.795 & 0.537 \\
\hline na5 & & & & 0.449 & 0.000 & 0.000 & -0.055 & & -0.055 & 0.394 & 0.291 \\
\hline na6 & & & & 0.553 & 0.000 & 0.000 & 0.000 & & 0.000 & 0.553 & 0.329 \\
\hline
\end{tabular}

Note: Expected influence of nodes within the same dimension (within-EI) and between other dimensions (between-EI), bridge expected influence (bridge-EI), global expected influence (EI), and nodes predictability $\left(\mathrm{R}^{2}\right)$. 
Flourishing cross-dimensional connections. Bridge-EI metrics showed that positive relationships (f2), social contribution (f4) and competence (f5) did not exhibited direct connections with the other well-being dimensions. By means of between-EI metrics the cross-dimensional connections of the FS items can be partitioned as follows: Meaning in life (f1) showed direct, positive connections with all the other three well-being dimensions; Engagement (f3) was connected to LS and PA, but not with NA; Self-acceptance (f6) was positively associated with LS and negatively with NA; Optimism (f7) showed the strongest cross-dimensional connections, positive ones with LS and PA, and negative with NA; Finally, being respected (f8) was directly connected exclusively with LS.

A worth mentionable specific pattern of connections emerged between f1 ("I lead a purposeful and meaningful life") and affect indicators. Having purpose and meaning in life was positively associated with pal ("positive") and pa6 ("negative"), but also to na1 ("negative"). The association between $\mathrm{f} 1$ and nal became negative when it was mediated by $\mathrm{f} 7$ ("I am optimistic about my future"), thus, it can be interpreted as if without a positive view of the future, leading a purposeful life is not a guarantee to avoid experiencing negative emotions.

Subjective Well-Being components within and between expected influence. The cognitive component of SWB, namely LS, exhibited strong interconnections within its indicators, with withinEI indices ranging between .556 (1s5) and 1.126 (ls3). It is worth noting that whereas bridge-EI showed that all the LS items had an influence on flourishing, PA and NA taken together, between-EI disclosed their specific contribute on the separate domains. The first three SWLS items, focused on present LS, were connected to flourishing, but not to PA, while the last two SWLS item, focused on past LS, were connected to PA, but not to flourishing: Ideal life (ls1) was directly connected to purpose in life (f1), optimism (f7), and being respected (f8); Excellent life conditions (ls2) was tied to self-acceptance (f6); Satisfaction with life (ls3) was positively connected to engagement (f3) and optimism (f7), and negatively connected to "Sad" (na4); LS items taking as a term of comparison past life, namely ls4 and 1s5, were respectively connected only to "Contented" (pa6) and "Happy" (pa4). 
Thus, although LS has been frequently considered as a hedonic component, its connections toward flourishing were stronger than its connections toward PA and NA.

The PA and NA affective components showed high within-EI indices and were pretty tied to each other, with PA between-EI on NA ranging from -.300 to -.021, and with NA between-EI on PA ranging from -.339 to 0. Notably, the connections between the three general SPANE-P items and the three general SPANE-N items were stronger than the connections between the three specific SPANEP items and the three specific SPANE-N items, suggesting that the separability between PA and NA was mainly due to specific, rather than general, emotional contents. General affect items such as positive (pa1), negative (na1) and bad (na2) were more related to flourishing, while specific affect items such as happy (pa4) and sad (na4) were more related to LS. The node pa6 (contented) was influent both on flourishing (between-EI $=.158$ ) and on LS (between-EI $=.053$ ).

\section{Discussions and conclusions}

This paper examined the validity of a quadripartite model of well-being by evaluating the role of flourishing as compared to the Diener's tripartite model of SWB. By means of network psychometrics it was possible to extract from a single EBIC GLASSO model several information that ranged from the dimensionality of the constructs under study to the specific relationships among the observed variables.

From a visual inspection of the estimated network, the well-being indicators appeared as clearly grouped in distinct nodes communities. Contrary to the theoretical conjecture that the separability among well-being dimensions can be grounded on a hedonic and eudaimonic distinction (Deci \& Ryan, 2008; Joshanloo, 2016), EGA results pointed to a categorization of the well-being indicators based on their type of content, that is global evaluation of life satisfaction, positive and negative affective experiences, and perceived positive functioning in life. With respect to the tripartite model of SWB, flourishing indicators were linked to each other to form their own topology, but at the same time they showed several connections both with the affective and the cognitive components. 
In contrast to the perspectives that incorporate positive emotions in the flourishing definition (Keyes, 2002; Seligman, 2011), our results suggest that the strength of the connections between flourishing and PA was not preponderant compared to the strength of the connections between flourishing and LS. Whether on the one hand PA indicators stimulate respondents to explicitly formulate their evaluations on the basis of their affective experiences, on the other hand flourishing indicators, as well as LS indicators, may involve affective information only on a less explicit level. Thus, it seems natural to separately measure well-being components that convey different sources of information.

Another point of interest regarding the items' semantic refers to their hierarchy. Both flourishing and LS can be conceived as subjective cognitive evaluations, but whereas the former relies on perceived positive functioning in specific areas of life (e.g., relationships, self-acceptance), the latter is located at a superior level given the global nature of the evaluation. The analyses of nodes' importance showed that the item with the highest value of EI as well with the highest predictability $\left(\mathrm{R}^{2}=.75\right)$, was "I am satisfied with my life". Indeed, this item represents the most general level of abstraction that can be achieved as an indicator of subjective well-being, likely embedding both affective experience and perceived functioning as benchmarks to build one's own global evaluation.

Although the validation studies on the FS confirmed its one-dimensional structure, and also the EGA in the present study identified all the FS items as pertaining to the same dimension, the content of the items clearly reflects the subjective perception of positive functioning in different areas of life. The effect of the different semantic content and semantic hierarchy of the FS items can be easily observed in the network model. Indeed, FS items concerning positive evaluations upon functioning in life in general (i.e., positive view of the future, engagement in daily activities, purpose in life) were closer and directly connected to LS, whereas FS items describing more specific area of life, as those referred to social well-being, were more distant and indirectly connected to LS.

A strength of the present study was the use of a novel methodological approach to the study of the well-being structure. Unlike confirmatory factor models in which the interconnections among the specific indicators are neglected due to the local independence assumption, network modeling 
allows to evaluate the separate contribution of the indicators to the model and the specific interrelations among them. The well-being structure can thus be derived from a data-driven approach in which the separability among dimensions can emerge directly from the relationships among the items themselves without the need to advocate latent factors.

Furthermore, we proposed two new indices to evaluate nodes' importance in multidimensional networks, namely within-EI and between-EI. The specific contribution of a node within its dimension is useful to evaluate the internal consistency of a specific scale, whereas specific crossdimensional pattern of connections can be summarized by the between-EI metrics. A precise functional description of the strength centrality decomposition may be very important in understanding network structure and will be deferred to future analysis.

Limitations of this study are as follows. First, our results are based on an Italian adult sample, whereas it should be desirable to test the network quadripartite Diener's model across life-span and in different cultures. Second, it should be interesting to extend the well-being network model including other flourishing indicators, such as autonomy and personal growth (Ryff, 1989), and other specific affect indicators such as calm and serenity that are deemed particularly important in eastern Asian cultures (Tsai et al., 2006). Third, the cross-sectional nature of the design prevented us to provide any causal interpretation on the reciprocal influence among well-being facets. Future research should explore longitudinal within-person data (e.g., Daily Reconstruction Method; Anusic, Lucas, \& Donnellan, 2017; Kahneman, Krueger, Schkade, Schwarz, \& Stone, 2004). In such a full prospective design, potential causal paths connecting flourishing with PA, NA and LS could be tested, as well the stability of the different dimensions of well-being over time. Furthermore, individual network structures may reveal whether differences in network connectivity are also found at the level of the individuals.

To conclude, the study of well-being through network modeling may yield several new possibilities to go beyond the conventional classification of hedonic and eudaimonic dimensions. In the last decade there was a drive to integrate both the hedonic and the eudaimonic concepts of well- 
being based on the idea that the distinction between two qualitatively different types of well-being is merely a theoretical artifice (Kashdan, Biswas-Diener, \& King, 2008). Coherently with this idea, our findings show that SWB components, on the one side, and flourishing, on the other, despite belonging to different theoretical traditions are indeed closely related constructs that influence each other and together conspire in the rise of well-being.

\section{Supplementary materials}

All relevant data and materials can be found at:

https://osf.io/gj7d6/?view_only=a33209b42f0448ad9b51ded68647189d

\section{References}

Anusic, I., Lucas, R. E., \& Donnellan, M. B. (2017). The Validity of the Day Reconstruction Method in the German Socio-economic Panel Study. Social Indicators Research, 130(1), 213-232. doi:10.1007/s11205-015-1172-6

Bollen, K.A. (1989). Structural Equations with Latent Variables. New York: John Wiley \& Sons. doi:10.1002/9781118619179

Bradburn, N. M. (1969). The structure of psychological well-being. Chicago: Aldine

Busseri, M. A., \& Sadava, S. W. (2010). A Review of the Tripartite Structure of Subjective WellBeing: Implications for Conceptualization, Operationalization, Analysis, and Synthesis. Personality and Social Psychology Review, 15(3), 290-314. doi:10.1177/1088868310391271

Campbell, A., Converse, P. E., \& Rodgers, W. L. (1976). The quality of American life. New York: Russell Sage Foundation. 
Chandrasekaran, V., Parrilo, P. A., \& Willsky, A. S. (2012). Latent variable graphical model selection via convex optimization. The Annals of Statistics, 40(4), 1935-1967. doi:10.1214/11-aos949

Chen, J., \& Chen, Z. (2008). Extended Bayesian information criteria for model selection with large model spaces. Biometrika, 95(3), 759-771. doi:10.1093/biomet/asn034

Compton, W. C., Smith, M. L., Cornish, K. A., \& Qualls, D. L. (1996). Factor structure of mental health measures. Journal of Personality and Social Psychology, 71(2), 406-413. doi:10.1037/0022-3514.71.2.406

Costa, P. T., \& McCrae, R. R. (1980). Influence of extraversion and neuroticism on subjective wellbeing: Happy and unhappy people. Journal of Personality and Social Psychology, 38(4), 668-678. doi:10.1037/0022-3514.38.4.668

Deci, E. L., \& Ryan, R. M. (2000). The "What" and "Why" of Goal Pursuits: Human Needs and the Self-Determination of Behavior. Psychological Inquiry, 11(4), 227-268. doi:10.1207/s15327965pli1104_01

Deci, E. L., \& Ryan, R. M. (2008). Hedonia, eudaimonia, and well-being: an introduction. Journal of Happiness Studies, 9(1), 1-11. doi:10.1007/s10902-006-9018-1

Diener, E. (1984). Subjective well-being. Psychological Bulletin, 95(3), 542-575. doi:10.1037/0033-2909.95.3.542

Diener, E. (1994). Assessing subjective well-being: Progress and opportunities. Social Indicators Research, 31(2), 103-157. doi:10.1007/bf01207052

Diener, E. (2006). Guidelines for National Indicators of Subjective Well-Being and Ill-Being. Applied Research in Quality of Life, 1(2), 151-157. doi:10.1007/s11482-006-9007-x

Diener, E. (2008). Myths in the science of happiness, and directions for future research. In M. Eid \& R. J. Larsen (Eds.), The science of subjective well-being (pp. 493-514). New York, NY: Guilford. 
Diener, E., \& Emmons, R. A. (1984). The independence of positive and negative affect. Journal of Personality and Social Psychology, 47(5), 1105-1117. doi:10.1037/0022-3514.47.5.1105

Diener, E., \& Lucas, R. E. (2000). Explaining differences in societal levels of happiness: Relative standards, need fulfillment, culture, and evaluation theory. Journal of Happiness Studies, l(1), 41-78. doi: 10.1023/A:1010076127199

Diener, E., Colvin, C. R., Pavot, W. G., \& Allman, A. (1991). The psychic costs of intense positive affect. Journal of Personality and Social Psychology, 61(3), 492-503. doi:10.1037/00223514.61.3.492

Diener, E., Emmons, R. A., Larsen, R. J., \& Griffin, S. (1985). The Satisfaction With Life Scale. Journal of Personality Assessment, 49(1), 71-75. doi:10.1207/s15327752jpa4901_13

Diener, E., Suh, E. M., Lucas, R. E., \& Smith, H. L. (1999). Subjective well-being: Three decades of progress. Psychological Bulletin, 125(2), 276-302. doi:10.1037/0033-2909.125.2.276

Diener, E., Wirtz, D., Tov, W., Kim-Prieto, C., Choi, D., Oishi, S., \& Biswas-Diener, R. (2010). New Well-being Measures: Short Scales to Assess Flourishing and Positive and Negative Feelings. Social Indicators Research, 97(2), 143-156. doi:10.1007/s11205-009-9493-y

Disabato, D. J., Goodman, F. R., Kashdan, T. B., Short, J. L., \& Jarden, A. (2016). Different types of well-being? A cross-cultural examination of hedonic and eudaimonic well-being. Psychological Assessment, 28(5), 471-482. doi:10.1037/pas0000209

Easterlin, R. A. (1974). Does economic growth improve the human lot? Some empirical evidence. In P. A. David \& M. W. Reder (Eds.), Nations and households in economic growth (pp. 89125). New York: Academic Press. doi:10.1016/b978-0-12-205050-3.50008-7

Epskamp, S. (2019). bootnet: Bootstrap methods for various network estimation routines. Version 1.2.2. https://cran.r-project.org/web/packages/bootnet/

Epskamp, S., \& Fried, E. I. (2018). A tutorial on regularized partial correlation networks. Psychological Methods, 23(4), 617-634. doi:10.1037/met0000167 
Epskamp, S., Cramer, A. O. J., Waldorp, L. J., Schmittmann, V. D., \& Borsboom, D. (2012). qgraph: Network Visualizations of Relationships in Psychometric Data. Journal of Statistical Software, 48(4). doi:10.18637/jss.v048.i04

Epskamp, S., Maris, G., Waldorp, L. J., \& Borsboom, D. (2018). Network Psychometrics. The Wiley Handbook of Psychometric Testing, 953-986. doi:10.1002/9781118489772.ch30

Epskamp, S., Rhemtulla, M., \& Borsboom, D. (2017). Generalized Network Psychometrics: Combining Network and Latent Variable Models. Psychometrika, 82(4), 904-927. doi:10.1007/s11336-017-9557-x

Foygel, R., \& Drton, M. (2010). Extended Bayesian information criteria for Gaussian graphical models. Advances in neural information processing systems, 23, 20200-2028. arXiv:1011.6640

Friedman, J., Hastie, T., \& Tibshirani, R. (2008). Sparse inverse covariance estimation with the graphical lasso. Biostatistics, 9(3), 432-441. doi:10.1093/biostatistics/kxm045

Giuntoli, L., Ceccarini, F., Sica, C., \& Caudek, C. (2017). Validation of the Italian Versions of the Flourishing Scale and of the Scale of Positive and Negative Experience. SAGE Open, 7(1), 215824401668229. doi:10.1177/2158244016682293

Golino, H. F., \& Demetriou, A. (2017). Estimating the dimensionality of intelligence like data using Exploratory Graph Analysis. Intelligence, 62, 54-70. doi:10.1016/j.intell.2017.02.007

Golino, H. F., \& Epskamp, S. (2017). Exploratory graph analysis: A new approach for estimating the number of dimensions in psychological research. PLOS ONE, 12(6), e0174035. doi:10.1371/journal.pone.0174035

Golino, H. F., \& Epskamp, S. (2017). Exploratory graph analysis: A new approach for estimating the number of dimensions in psychological research. PLOS ONE, 12(6), e0174035. doi:10.1371/journal.pone.0174035 
Goodman, F. R., Disabato, D. J., Kashdan, T. B., \& Kauffman, S. B. (2018). Measuring well-being: A comparison of subjective well-being and PERMA. The Journal of Positive Psychology, 13(4), 321-332. doi:10.1080/17439760.2017.1388434

Haslbeck, J. M. B., \& Waldorp, L. J. (2018). How well do network models predict observations?

On the importance of predictability in network models. Behavior Research Methods, 50(2), 853-861. doi:10.3758/s13428-017-0910-x

Haslbeck, J. M., \& Waldorp, L. J. (2016). mgm: Structure Estimation for Time-Varying Mixed Graphical Models in high-dimensional Data. arXiv preprint:1510.06871v2 http://arxiv.org/abs/1510.06871v2.

Hone, L., Jarden, A., \& Schofield, G. (2013). Psychometric Properties of the Flourishing Scale in a New Zealand Sample. Social Indicators Research, 119(2), 1031-1045. doi:10.1007/s11205013-0501-X

Huppert, F. A., \& So, T. T. C. (2013). Flourishing Across Europe: Application of a New Conceptual Framework for Defining Well-Being. Social Indicators Research, 110(3), 837861. doi:10.1007/s11205-011-9966-7

Jones, P.J., 2017. Networktools: Tools for Identifying Important Nodes in Networks. R Package Version 1.2.0. https://CRAN.R-project.org/package=networktools Joshanloo, M. (2016). Revisiting the Empirical Distinction Between Hedonic and Eudaimonic Aspects of Well-Being Using Exploratory Structural Equation Modeling. Journal of Happiness Studies, 17(5), 2023-2036. doi:10.1007/s10902-015-9683-z

Jovanović, V. (2015). Beyond the PANAS: Incremental validity of the Scale of Positive and Negative Experience (SPANE) in relation to well-being. Personality and Individual Differences, 86, 487-491. doi:10.1016/j.paid.2015.07.015

Kahneman, D., Diener, E., \& Schwarz, N. (Eds.). (1999). Well-being: The Foundations of hedonic psychology. New York: Russell Sage Foundation. 
Kahneman, D., Krueger, A. B., Schkade, D. A., Schwarz, N., \& Stone, A. A. (2004). A survey method for characterizing daily life experience: The day reconstruction method. Science, 306(5702), 1776-1780. doi:10.1126/science.1103572.

Kashdan, T. B., Biswas-Diener, R., \& King, L. A. (2008). Reconsidering happiness: the costs of distinguishing between hedonics and eudaimonia. The Journal of Positive Psychology, 3(4), 219-233. doi:10.1080/17439760802303044

Keith, T. Z., Caemmerer, J. M., \& Reynolds, M. R. (2016). Comparison of methods for factor extraction for cognitive test-like data: Which overfactor, which underfactor? Intelligence, 54, 37-54. doi:10.1016/j.intell.2015.11.003

Keyes, C. L. M. (2002). The Mental Health Continuum: From Languishing to Flourishing in Life. Journal of Health and Social Behavior, 43(2), 207. doi:10.2307/3090197

Keyes, C. L. M., Shmotkin, D., \& Ryff, C. D. (2002). Optimizing well-being: The empirical encounter of two traditions. Journal of Personality and Social Psychology, 82(6), 10071022. doi:10.1037/0022-3514.82.6.1007

Linley, P. A., Maltby, J., Wood, A. M., Osborne, G., \& Hurling, R. (2009). Measuring happiness: The higher order factor structure of subjective and psychological well-being measures. Personality and Individual Differences, 47(8), 878-884. doi:10.1016/j.paid.2009.07.010

Lucas, R. E., Diener, E., \& Suh, E. (1996). Discriminant validity of well-being measures. Journal of Personality and Social Psychology, 71(3), 616-628. doi:10.1037/0022-3514.71.3.616 Lyubomirsky, S., \& Lepper, H. S. (1999). Social Indicators Research, 46(2), 137-155. doi:10.1023/a:1006824100041

Pavot, W., \& Diener, E. (1993). Review of the Satisfaction With Life Scale. Psychological Assessment, 5(2), 164-172. doi:10.1037/1040-3590.5.2.164

Pons, P., \& Latapy, M. (2006). Computing Communities in Large Networks Using Random Walks. Journal of Graph Algorithms and Applications, 10(2), 191-218. doi:10.7155/jgaa.00124 
R Core Team (2019). R: a language and environment for statistical computing. Version 3.6.0.

Vienna: R Foundation for Statistical Computing.

Radloff, L. S. (1977). The CES-D Scale. Applied Psychological Measurement, 1(3), 385-401. doi:10.1177/014662167700100306

Robinaugh, D. J., Millner, A. J., \& McNally, R. J. (2016). Identifying highly influential nodes in the complicated grief network. Journal of Abnormal Psychology, 125(6), 747-757. doi:10.1037/abn0000181

Ryff, C. D. (1989). Happiness is everything, or is it? Explorations on the meaning of psychological well-being. Journal of Personality and Social Psychology, 57(6), 1069-1081. doi:10.1037/0022-3514.57.6.1069

Ryff, C. D., \& Keyes, C. L. M. (1995). The structure of psychological well-being revisited. Journal of Personality and Social Psychology, 69(4), 719-727. doi:10.1037/0022-3514.69.4.719

Schimmack, U. (2008). The structure of subjective well-being. In M. Eid, \& R. J. Larsen (Eds.). The science of subjective well-being (pp. 97-123). New York, NY: Guilford Press.

Seligman, M. (2011). Flourish: A visionary new understanding of happiness and well-being. New York: Simon \& Schuster.

Silva, A. J., \& Caetano, A. (2011). Validation of the Flourishing Scale and Scale of Positive and Negative Experience in Portugal. Social Indicators Research, 110(2), 469-478. doi:10.1007/s11205-011-9938-y

Slocum-Gori, S. L., Zumbo, B. D., Michalos, A. C., \& Diener, E. (2008). A Note on the Dimensionality of Quality of Life Scales: An Illustration with the Satisfaction with Life Scale (SWLS). Social Indicators Research, 92(3), 489-496. doi:10.1007/s11205-008-9303y

Tov, W., \& Diener, E. (2007). Culture and subjective well-being. In S. Kitayama \& D. Cohen (Eds.), Handbook of cultural psychology (pp. 691-713). New York, NY: Guilford. 
Tsai, J. L., Knutson, B., \& Fung, H. H. (2006). Cultural variation in affect valuation. Journal of Personality and Social Psychology, 90(2), 288-307. doi:10.1037/0022-3514.90.2.288

Waterman, A. S. (1993). Two conceptions of happiness: Contrasts of personal expressiveness (eudaimonia) and hedonic enjoyment. Journal of Personality and Social Psychology, 64(4), 678-691. doi:10.1037/0022-3514.64.4.678

Watson, D., Clark, L. A., \& Tellegen, A. (1988). Development and validation of brief measures of positive and negative affect: The PANAS scales. Journal of Personality and Social Psychology, 54(6), 1063-1070. doi:10.1037/0022-3514.54.6.1063

Wilson, W. R. (1967). Correlates of avowed happiness. Psychological Bulletin, 67(4), 294-306. doi:10.1037/h0024431 